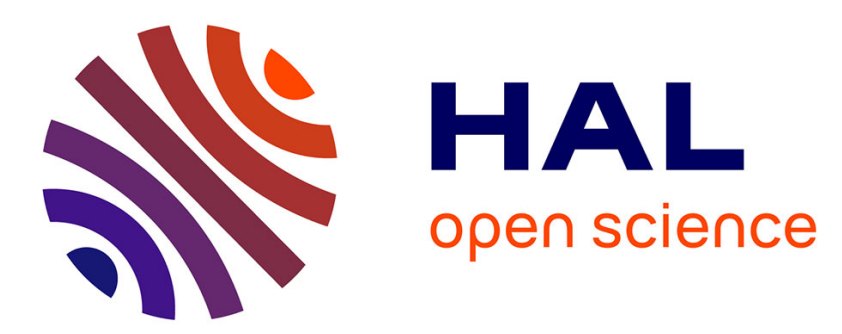

\title{
Optimal composition of number and size of machines in a multi-stage make-to-order system with due dates
}

\author{
Klaus Altendorfer, Stefan Minner
}

\section{To cite this version:}

Klaus Altendorfer, Stefan Minner. Optimal composition of number and size of machines in a multistage make-to-order system with due dates. International Journal of Production Research, 2011, 10.1080/00207543.2010.543940 . hal-00711442

\section{HAL Id: hal-00711442 \\ https://hal.science/hal-00711442}

Submitted on 25 Jun 2012

HAL is a multi-disciplinary open access archive for the deposit and dissemination of scientific research documents, whether they are published or not. The documents may come from teaching and research institutions in France or abroad, or from public or private research centers.
L'archive ouverte pluridisciplinaire HAL, est destinée au dépôt et à la diffusion de documents scientifiques de niveau recherche, publiés ou non, émanant des établissements d'enseignement et de recherche français ou étrangers, des laboratoires publics ou privés. 


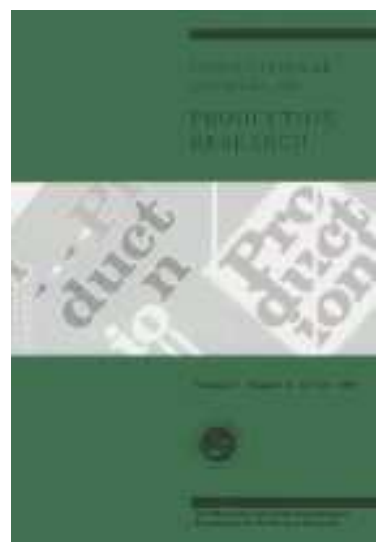

\section{Optimal composition of number and size of machines in a multi-stage make-to-order system with due dates}

\begin{tabular}{|r|l|}
\hline Journal: & International Journal of Production Research \\
\hline Manuscript ID: & TPRS-2010-IJPR-0576.R1 \\
\hline Manuscript Type: & Original Manuscript \\
\hline Aute Submitted by the \\
Author: & $13-$ Sep-2010 \\
\hline Keywote List of Authors: & $\begin{array}{l}\text { Altendorfer, Klaus; University of Applied Sciences Upper Austria, } \\
\text { School of Management } \\
\text { Minner, Stefan; University of Vienna, Department of Business } \\
\text { Administration }\end{array}$ \\
\hline MAKE TO ORDER PRODUCTION, INVENTORY MANAGEMENT, \\
QUEUEING MODELS
\end{tabular}

\section{SCHOLARONE"}

Manuscripts 


\title{
Optimal composition of number and size of machines in a multi-stage make-to-order system with due dates
}

\author{
Klaus Altendorfer ${ }^{\mathrm{a}}$, Stefan Minner ${ }^{\mathrm{b}}$ \\ ${ }^{a}$ Department of Operations Management, School of Management, Upper Austrian \\ University of Applied Sciences, A-4400 Steyr, Austria \\ ${ }^{b}$ Department of Business Administration, University of Vienna, A-1210 Vienna, \\ Austria \\ e-mail: klaus.altendorfer@fh-steyr.at, \\ telephone: $+43 / 7252 / 884-3150$ \\ fax: $+43 / 7252 / 884-3199$
}

\begin{abstract}
We develop an optimization model to minimize costs for work-in-process (WIP), finished goods inventory (FGI), backorders, and capacity for a multi-stage production system applying a work ahead window work release policy. Customers arrive to a maketo-order production system and have stochastic due dates. The parameters to optimize are the capacity invested at each stage, consisting of the number of machines and the processing rate (defined by a set of possible processing rates), as well as the work ahead window. An optimality condition is provided showing when it is optimal to invest into a single machine at each stage if processing rates are continuous decision variables. The optimality of increasing capacity towards the customer end of the production line is proven under certain conditions. For a special case consisting of two stages with M/M/s queues with exponentially distributed customer required lead time, explicit expressions for WIP, FGI and backorders are developed. A set of numerical examples illustrates the influence of predefined processing rates on the optimal number and selection of machines. A simple solution heuristic for the problem with a predefined set of processing rates is proposed and its performance is shown in a numerical example. Additionally, the influence of uncertain input rates into the production system is discussed.
\end{abstract}

Key words: make-to-order manufacturing, inventory, capacity investment, queuing, stochastic due dates

\section{Introduction}

The design of manufacturing and service operations requires an adequate installation

of capacity and the selection and specification of operating policies. In this rich field

of research, queuing models are widely used to support such decisions and

performance evaluations. Capacity can be installed mainly through two decisions, the number and the throughput of servers both affecting total capacity. Hereby, an important trade-off exists between the number and size of machines, i.e. a few large versus a larger number of small machines. The complexity of such a tactical decision further increases in a multi-stage system where capacity levels between stages need to be appropriately coordinated and costs for investments need to be further traded-off 
with inventory and backorder costs whereby decisions need to correctly anticipate operational replenishment and workload control decisionsIn the majority of the available literature, processing rates can be set as continuous variables. In reality, however, there often exists a finite set of possible options which can be expressed as a predefined set of possible processing rates. The finiteness of manufacturing rates can be motivated either by available technologies, e.g. a machine can only run at certain speeds, requires certain batch sizes, etc. or by restrictions imposed by the workforce. Regulations might require that a worker has to be compensated for a minimum number of hours and that working time is organized in full shifts (either 1, 2, or 3 per day) or a certain number of full working days. Furthermore, different workforce qualifications and the technology of automatic handling systems can also lead to a predefined set of processing rates.

If customer orders have a random due date, production can be organized according to a make-to-order (MTO) policy whereby the work release to the production system can be constrained with a work ahead window policy. The work ahead window states that an order is only released to the production system when its remaining time to due date is shorter than the work ahead window (see also Hopp and Spearman, 1996 or Jodlbauer, 2008 for details). The question of which technology option to choose and how much machines to invest into for minimizing overall costs in such a system is one continuously faced by production managers and therefore of practical relevance. For this reason, we investigate the influence of a predefined set of processing rates on the optimal capacity investment in an MTO production system with stochastic due dates, a work ahead window work release policy, and include finished-goods-inventory (FGI) and backorder costs. The resulting research questions are: 
1) Under which conditions is a single machine policy at each stage optimal in an $n$-stage serial MTO production system with stochastic due dates?

2) What is the relationship between optimal capacity levels at consecutive levels in a multi-stage system?

3) What is the cost penalty of investing into more than one machine at each stage when processing rates have a continuous range?

4) What is the impact of predefined processing rates on overall costs in an MTO production system?

5) What is the impact of demand parameter uncertainty on capacity design?

The first two questions will be investigated analytically for the general $n$-stage serial manufacturing system whereas the remaining three questions will be answered numerically and we therefore restrict the analysis to a two-stage M/M/s system and exponentially distributed due dates.

This manuscript extends Altendorfer and Minner, (2010) who show that the optimal planned lead time in a two-stage MTO production system is independent of the customer required lead time and that only a marginal cost increase is incurred if a work ahead window policy is applied instead of a planned lead time at each stage. Building on these results, we address the trade-off between multiple machines and machine sizes and here especially on a predefined set of technologies rather than only allowing for a single machine with continuous processing rate at each stage. Therefore, available queuing design models (for a review see Rao et al., (1998)) are extended by including random customer due dates, FGI as well as backorder costs, and workload is released to the system by a work ahead window policy.

A general condition for the single machine optimality at each stage is derived for an $n$-stage serial production system. We find that in a system with value added 
work-in-process (WIP) cost structure the distribution of customer required lead time has no influence on the property that processing rates should increase towards the customer end of the line. For continuous processing rates, there is a considerable cost penalty to pay if more than one machine is invested. However, if only a set of predefined processing rates is available, these predefined processing rates have a significant influence on optimal costs and a numerical study shows that in this case more than one machine is optimal in a considerable number of test instances. Relating these findings to the TPS (Toyota Production System) philosophy to invest into small and simple machines, which in TPS is mainly based on maintenance, flexibility and machine failure arguments, shows that for predefined processing rates the cost premium paid in TPS can be rather small or the small machines invested can even be cost optimal. This finding, which cannot be derived in a continuous processing rate model, supports the application of TPS from an investment cost perspective. The paper is structured as follows. Section 2 provides a review of the related literature. In Section 3 , the general $n$-stage model is introduced and expressions for WIP, FGI, and backorder costs for the two-stage M/M/s production system are developed in Section 4. Structural properties of optimal solutions are derived through a numerical example in Section 5 which illustrates the impact of predefined processing rates.

\section{Literature Review}

For make-to-stock (MTS) production systems, the optimal replenishment strategy minimizing inventory and backorder costs is a broadly discussed topic, see Silver, Pyke and Peterson, (1998) and Zipkin, (2000), both with an independent (exogenous) and dependent (endogenous) distribution of replenishment lead time. For MTO production systems, inventory balancing against backorder costs is discussed in Yano, (1987), Buzacott and Shanthikumar, (1994), Bertrand and van Ooijen, (2008), and 
Altendorfer and Minner (2010), all of which address the topic of optimal planned lead time or optimal work ahead window.

Crabill, Gross and Magazine, (1977), Buzacott and Shanthikumar, (1992) or more recently Rao et al., (1998) give an overview on the topic of queuing system design. Determining the optimal number and size of machines has been addressed in Brigham, (1955), Hillier, (1963), and Stidham, Jr., (1970) all stating conditions or queuing system specifications where single servers lead to the optimal capacity and waiting time costs. Brumelle, (1971), Driscoll and Weiss, (1976), Stidham, Jr., (1992), Mandelbaum and Reiman, (1998), Chao and Scott, (2000), Scheller-Wolf, (2003) and MacGregor-Smith, (2007) have focused on the optimal number of servers or optimal processing rates. These models mainly balance costs for waiting with costs of capacity. The majority finds that a single server queue leads to the optimal result when linear capacity costs and a continuous range of processing rate is assumed (see e.g. the fundamental paper of Stidham, Jr., 1970 for quite general conditions). Nevertheless, Mandelbaum and Reiman, (1998), Chao and Scott, (2000), SchellerWolf, (2003) and MacGregor-Smith, (2007) have also shown that for certain queue specifications or certain changes in the objective function, multiple servers could be optimal.

Literature including backorder costs or a service level constraint in addition to the capacity and waiting time costs while optimizing the capacity investment for MTO and MTS manufacturing systems, is Angelus and Porteus, (2002), Bradley and Glynn, (2002), Raman and Kim, (2002), Mincsovics, Tan and Alp, (2009), and Jodlbauer and Altendorfer, (2010). Most of these contributions analyze single machine or single-stage production systems with continuous capacity investment possibilities. 


\section{General $n$-stage optimization problem}

\subsection{Model description and assumptions}

We assume a manufacturing system with $i=1,2, \ldots, n$ stages numbered in reverse order from $n$ (most upstream) to 1 (most downstream) as shown in Figure 1. Each stage $i$ has $s_{i}$ (identical) parallel machines ( $s_{i}$ being integer).

Customers arrive to the manufacturing system according to a stochastic process with mean arrival rate $\lambda$. Each customer requests an individual due date that determines the non-negative random customer required lead time $L$. Let $f_{L}(\cdot)$ denote the probability density function (pdf) of the customer required lead time, $F_{L}(\cdot)$ the respective cumulative distribution function (cdf), and $1 / \beta$ its mean. The customer required lead time cannot be influenced by the production system and all customer orders are accepted. Customer orders are processed consecutively by the $n$ stages of the manufacturing system following a first-in-first-served discipline. Each stage $i$ consists of one shared buffer for waiting orders and $s_{i}$ identical parallel machines with random processing rate with mean $\mu_{i}$ per unit of time of one machine at stage $i$. The parameter $\mu_{i}$ is assumed to be a decision variable restricted to a set of feasible values $\Omega$. The capacity at each stage is therefore given by $s_{i} \mu_{i}$ which is assumed to be determined by an investment decision either including both the processing rate and the machine number or any of the two single variables. $c_{\mu, i}$ denotes the capacity cost per unit of installed capacity at stage $i . W_{i}$ denotes the random variable of production lead time at stage $i$ needed for one order from arrival at stage $i$ until its completion. $\tilde{W}_{i}=\sum_{j=1}^{i} W_{j}$ denotes the remaining production lead time from arrival at stage $i$ until its delivery to the FGI buffer. Orders are released to stage $n$ of the production system whenever their remaining time to due date is lower than a predefined work ahead 
Insert Figure 1 about here.

Let $Y_{i}$ denote the random WIP at stage $i$ and $c_{y, i}$ the respective holding cost per order per unit of time stored at stage $i$. We assume a value added WIP holding cost structure, i.e. an increase after each processing step, $c_{y, i} \geq c_{y, i+1}$. Let $c_{y, n+1}=0$; i.e., holding costs for orders waiting in the order list before stage $n$ are neglected. The accounting of WIP at stage $i$ starts when the order is released to the buffer $i$ and ends when the order is delivered to buffer $i-1$. Let $G$ denote the random finished goods inventory and $c_{f}$ the respective inventory holding cost per order per unit of time. Backorders $B$ are charged a backorder $\operatorname{cost} c_{c}$ per unit of time an order is late. $C$ denotes the tardiness random variable of an order and $I$ the random FGI lead time. Minimizing expected WIP, FGI, backorder and capacity costs leads to optimization problem (1).

$$
\begin{aligned}
& \sum_{i=1}^{n} E\left[\tilde{W}_{i}\right] \lambda\left(c_{y, i}-c_{y, i+1}\right)+E[G] c_{f}+E[B] c_{c}+\sum_{i=1}^{n} s_{i} \mu_{i} c_{\mu, i} \rightarrow \min _{X,\left\{s_{i}\right\},\left\{\mu_{i}\right\}} \\
& s_{i} \mu_{i}>\lambda \forall i \text { and } s_{i} \in N^{+}
\end{aligned}
$$

The WIP cost calculation in equation (1) is based on the relative WIP costs added per stage. The second line of (1) is the stability condition stating that utilization at each stage has to be lower than $100 \%$. 


\section{2. $\quad$ Model analysis}

For a single stage as well as a serial production system, expressions for expected FGI and backorders without stochastic due dates are provided by Buzacott and Shanthikumar, (1994) and Yano, (1987). Altendorfer and Jodlbauer, (2010) and Altendorfer and Minner, (2010) extend these equations to include a random due date as follows:

$$
\begin{aligned}
& E[I]=\int_{X}^{\infty} \int_{0}^{X} f_{W}(\tau)(X-\tau) d \tau f_{L}(\theta) d \theta+\int_{0}^{X} \int_{0}^{\theta} f_{W}(\tau)(\theta-\tau) d \tau f_{L}(\theta) d \theta \\
& =\int_{0}^{X} F_{W}(\tau)\left(1-F_{L}(\tau)\right) d \tau \\
& E[C]=\int_{X}^{\infty} \int_{X}^{\infty} f_{W}(\tau)(\tau-X) d \tau f_{L}(\theta) d \theta+\int_{0}^{X} \int_{\theta}^{\infty} f_{W}(\tau)(\tau-\theta) d \tau f_{L}(\theta) d \theta \\
& =E[W]-\int_{0}^{X}\left(1-F_{W}(\tau)\right)\left(1-F_{L}(\tau)\right) d \tau=E[I]+E[W]-\int_{0}^{X}\left(1-F_{L}(\tau)\right) d \tau
\end{aligned}
$$

From the definition of the remaining production lead time $\left(\tilde{W}_{i}=\sum_{j=1}^{i} W_{j}\right)$ it follows that the pdf of $\tilde{W}_{i}$ is a convolution of the pdfs of all remaining production lead time values $W_{j}$ with $1 \leq j \leq i$. Denoting the convolution between the pdf of stochastic variable $\tilde{W}_{i-1}$ with the pdf of $W_{i}$ with $\int_{0}^{t} f_{\tilde{W}_{i-1}}(\tau) f_{W_{i}}(t-\tau) d \tau=f_{\tilde{W}_{i-1}}(t) * f_{W_{i}}(t)$ leads to:

$$
f_{\tilde{W}_{i}}(t)=\left(\left(\left(f_{W_{1}}(t) * f_{W_{2}}(t)\right) * f_{W_{3}}(t)\right) * \ldots * f_{W_{i}}(t)\right)
$$

For the general optimization problem, the following Proposition concerning the single machine optimality can be stated.

Proposition 1: For any manufacturing system consisting of a series of processing stages with parallel machines defined by $\left(\left\{s_{i}\right\} ;\left\{\mu_{i}\right\}\right)$ and continuous processing rates, a single machine policy $\left(\{1\} ;\left\{\mu_{i}^{1}\right\}\right)$ is optimal to minimize WIP, FGI, backorder and capacity costs as defined in equation (1) when the remaining production lead 
time of the parallel machine system at each stage second order stochastic dominates the remaining production lead time of the single machine stage (see condition (5)).

$$
\int_{0}^{\theta} F_{\tilde{W}_{i}^{1}}(\tau)-F_{\tilde{W}_{i}}(\tau) d \tau \geq 0 \forall \theta>0 \text { and } \exists \theta: \int_{0}^{\theta} F_{\tilde{W}_{i}^{1}}(\tau)-F_{\tilde{W}_{i}}(\tau) d \tau>0 \quad \forall i
$$

Proof see Appendix.

For a single stage $\mathrm{M} / \mathrm{M} / \mathrm{s}$ queuing system without considering a distribution of customer required lead time, Stidham Jr. (1970) shows that condition (5) is fulfilled and therefore the optimality of a single server. However, this does not hold in general. Brumelle (1971) provides an example for a squared coefficient of variation of the processing time greater than 1 where condition (5) is not fulfilled. However, the definition in equation (4) shows that the condition is fulfilled for a series of $\mathrm{M} / \mathrm{M} / \mathrm{s}$ queues as discussed in the next sections. Proposition 1 is intuitive for a series of $\mathrm{M} / \mathrm{M} / \mathrm{s}$ queues since the single machine at each stage is exactly tailored to the customer order rate.

Proposition 2: For any manufacturing system consisting of a series of processing stages with single machines and capacity costs increasing in processing rate $\left(C\left(\left\{\mu_{i}\right\}\right)=\sum_{i=1}^{n} \xi\left(\mu_{i}\right) c_{\mu, i}\right.$ and $\left.\frac{d \xi\left(\mu_{i}\right)}{d \mu_{i}}>0\right)$ and non-increasing in $\operatorname{stage}\left(c_{\mu, i} \geq c_{\mu, i-1}\right)$, the optimal processing rate is non-decreasing towards the FGI $\left(\mu_{i}^{*} \geq \mu_{i+1}^{*}\right)$ when WIP, FGI, backorder and capacity costs are minimized. Proof see Appendix.

A managerial implication of Proposition 2 is that (if not overruled by sufficiently increasing capacity costs) it is always better to have a higher downstream capacity and that the integration of a customer required lead time distribution has no influence on that property. 
Proposition 3: For any manufacturing system consisting of a series of processing stages with parallel machines, equal processing rates and linear capacity costs with respect to the number of machines at each stage $\left(C\left(\left\{s_{i}\right\}\right)=\sum_{i=1}^{n} s_{i} \mu c_{\mu, i}\right)$ which are non-increasing in stage $\left(c_{\mu, i} \geq c_{\mu, i-1}\right)$, the optimal allocation of machines is nondecreasing towards the FGI $\left(s_{i}^{*} \geq s_{i+1}^{*}\right)$ when WIP, FGI, backorder and capacity costs are minimized.

The proof is similar to the one for Proposition 2 and therefore omitted.

Proposition 3 shows that higher capacities should be kept towards the customer end of the production line not only for continuous processing rates but also for cases with a predefined machine size.

Proposition 4: For a manufacturing system consisting of a series of processing stages with parallel machines defined by $\left(\left\{s_{i}\right\} ;\left\{\mu_{i}\right\}\right)$ the cost for inventory, backorder and capacity as defined in equation (1) with $c_{y, k}=c_{f}=c_{y}$ are unimodal in $s_{i}$. Proof see Appendix.

Assuming that the condition $c_{y, k}=c_{f}=c_{y}$ has only minor influence on the unimodality and is rather a technical necessity for the proof, Proposition 4 can be applied to find a solution heuristic for the general optimization problem restricted to a predefined set of processing rates where $c_{y, k} \neq c_{f}$.

The general form of the optimization problem as stated in this section can be applied to find optimal parameters for any kind of $n$-stage production system as long as the production lead time distributions for the $n$ stages as well as the customer required lead time distribution are known. In the next section a solution heuristic for such a problem in a two-stage $\mathrm{M} / \mathrm{M} / \mathrm{s}$ production system is developed. 


\section{Optimization problem for a two-stage $\mathrm{M} / \mathrm{M} / \mathrm{s}$ production system}

In this section a special case with two stages, $\mathrm{M} / \mathrm{M} / \mathrm{s}$ queues, exponentially distributed customer required lead time and a predefined set of processing rates $\Omega$ is discussed. This two-stage setting, chosen for simplicity of exposition, allows for closed form expressions. However, by convolution of production lead time distributions, this could be extended to general serial systems.

Following Altendorfer and Jodlbauer, (2010), the implementation of a work ahead window in front of the production system modelled as $M / M / 1$ queue still leads to a Poisson input stream into the production stage for an exponentially distributed customer required lead time, such that all properties of an $M / M / 1$ queue remain valid. Since the M/M/s queue still maintains a Poisson output stream (see Tijms, 2003) all equations developed in this section are exact.

The optimization problem (1) simplifies to:

$$
\begin{aligned}
& E\left[W_{1}\right] \lambda\left(c_{y, 1}+c_{c}\right)+E\left[W_{2}\right] \lambda\left(c_{y, 2}+c_{c}\right)+E[I] \lambda\left(c_{f}+c_{c}\right) \\
& -\int_{0}^{X}\left(1-F_{L}(\tau)\right) d \tau \lambda c_{c}+s_{1} \mu_{1} c_{\mu, 1}+s_{2} \mu_{2} c_{\mu, 2} \rightarrow \min _{X, s_{1}, \mu_{1}, s_{2}, \mu_{2}} \\
& s_{1} \mu_{1}>\lambda \text { and } s_{2} \mu_{2}>\lambda ; \\
& \mu_{1}, \mu_{2} \in \Omega \text { and } s_{1}, s_{2} \in N^{+}
\end{aligned}
$$

To solve the optimization problem, the distributions $f_{\tilde{W}_{i}}(t)$ have to be calculated first. According to Medhi, (1991) the pdf of production lead time for an M/M/s queue can be stated as:

$$
\begin{aligned}
& f_{W_{M / M / s}}(t)=\sum_{i=0}^{s-1} \frac{(\rho s)^{i}}{i !} p_{0} \mu e^{-\mu t}+\frac{(\rho s)^{s}}{s !} p_{0} \frac{s \mu^{2}}{(s-1) \mu-\lambda}\left(e^{-\mu t}-e^{-(1-\rho) s \mu t}\right) \\
& p_{0}=\left[\sum_{i=0}^{s-1} \frac{(\rho s)^{i}}{i !}+\frac{(\rho s)^{s}}{s !(1-\rho)}\right]^{-1} ; \\
& \text { with } o=\frac{(\rho s)^{s}}{s !} p_{0} \frac{s \mu^{2}}{(s-1) \mu-\lambda} ; m=\sum_{i=0}^{s-1} \frac{(\rho s)^{i}}{i !} p_{0} \mu \\
& \Rightarrow f_{W_{M / M / s}}(t)=(m+o) e^{-\mu t}-o e^{-(1-\rho) s \mu t}
\end{aligned}
$$


$p_{0}$ is the probability that no order is in the production system and $\rho=\frac{\lambda}{s \mu}$ is the utilization of the production stage. $m$ and $o$ are defined as auxiliary variables for simplification of statements. Based on equation (7) it follows:

$$
\begin{aligned}
& f_{\tilde{W}_{1}}(t)=f_{W_{1}}(t)=\left(m_{2}+o_{2}\right) e^{-\mu t}-o_{2} e^{-(1-\rho) s \mu t} \\
& f_{W}(t)=f_{\tilde{W}_{2}}(t)=f_{W_{1}}(t) * f_{W_{2}}(t)=\int_{0}^{t} f_{W_{1}}(\tau) f_{W_{2}}(t-\tau) d \tau \\
& =\int_{0}^{t}\left(\left(m_{1}+o_{1}\right) e^{-\mu_{1} \tau}-o_{1} e^{-\left(1-\rho_{1}\right) s_{1} \mu_{1} \tau}\right)\left(\left(m_{2}+o_{2}\right) e^{-\mu_{2}(t-\tau)}-o_{2} e^{-\left(1-\rho_{2}\right) s_{2} \mu_{2}(t-\tau)}\right) d \tau \\
& =\left(m_{1}+o_{1}\right)\left(m_{2}+o_{2}\right) \frac{e^{-\mu_{1} t}-e^{-\mu_{2} t}}{\left(\mu_{2}-\mu_{1}\right)}-\left(m_{1}+o_{1}\right) o_{2} \frac{e^{-\left(s_{2} \mu_{2}-\lambda\right) t}-e^{-\mu_{1} t}}{\left(\mu_{1}-s_{2} \mu_{2}+\lambda\right)} \\
& -\left(m_{2}+o_{2}\right) o_{1} \frac{e^{-\left(s_{1} \mu_{1}-\lambda\right) t}-e^{-\mu_{2} t}}{\left(\mu_{2}-s_{1} \mu_{1}+\lambda\right)}+o_{1} o_{2} \frac{e^{-\left(s_{1} \mu_{1}-\lambda\right) t}-e^{-\left(s_{2} \mu_{2}-\lambda\right) t}}{\left(s_{2} \mu_{2}-s_{1} \mu_{1}\right)}
\end{aligned}
$$

For equations (8) to (12) non-identical processing rates are assumed. The equations for identical processing rates and identical number of machines are delivered in the Appendix.

\subsection{WIP, FGI and backorder calculation}

Using equation (8) leads to the following expected production lead time values:

$$
E\left[W_{i}\right]=\frac{\left(m_{i}+o_{i}\right)}{\mu_{i}^{2}}-\frac{o_{i}}{\left(\left(1-\rho_{i}\right) s_{i} \mu_{i}\right)^{2}}
$$

Applying equation (8), the cdf of the overall production lead time follows as:

$$
\begin{aligned}
& F_{W}(\tau)=\int_{0}^{\tau} f_{W}(\theta) d \theta=\left(t_{1}+t_{2}\right) \frac{1-e^{-\mu_{1} \tau}}{\mu_{1}}+\left(t_{3}-t_{1}\right) \frac{1-e^{-\mu_{2} \tau}}{\mu_{2}} \\
& -\left(t_{2}+t_{4}\right) \frac{1-e^{-\left(s_{2} \mu_{2}-\lambda\right) \tau}}{\left(s_{2} \mu_{2}-\lambda\right)}+\left(t_{4}-t_{3}\right) \frac{1-e^{-\left(s_{1} \mu_{1}-\lambda\right) \tau}}{\left(s_{1} \mu_{1}-\lambda\right)} \\
& \text { with } t_{1}=\frac{\left(m_{1}+o_{1}\right)\left(m_{2}+o_{2}\right)}{\left(\mu_{2}-\mu_{1}\right)} ; t_{2}=\frac{\left(m_{1}+o_{1}\right) o_{2}}{\left(\mu_{1}-s_{2} \mu_{2}+\lambda\right)} ; \\
& t_{3}=\frac{\left(m_{2}+o_{2}\right) o_{1}}{\left(\mu_{2}-s_{1} \mu_{1}+\lambda\right)} ; t_{4}=\frac{o_{1} o_{2}}{\left(s_{2} \mu_{2}-s_{1} \mu_{1}\right)}
\end{aligned}
$$

$t_{1}, t_{2}, t_{3}$ and $t_{4}$ are defined as auxiliary variables for simplification of statements.

With equation (2) this leads to the expected value for FGI lead time: 


$$
\begin{aligned}
& E[I]=\int_{0}^{X} F_{W}(\tau)\left(1-F_{L}(\tau)\right) d \tau \\
& =\left(\frac{\left(t_{1}+t_{2}\right)}{\mu_{1}}+\frac{\left(t_{3}-t_{1}\right)}{\mu_{2}}-\frac{\left(t_{2}+t_{4}\right)}{\left(s_{2} \mu_{2}-\lambda\right)}+\frac{\left(t_{4}-t_{3}\right)}{\left(s_{1} \mu_{1}-\lambda\right)}\right) \frac{1-e^{-\beta X}}{\beta} \\
& -\frac{\left(t_{1}+t_{2}\right)}{\mu_{1}} \frac{1-e^{-\left(\mu_{1}+\beta\right) X}}{\left(\mu_{1}+\beta\right)}-\frac{\left(t_{3}-t_{1}\right)}{\mu_{2}} \frac{1-e^{-\left(\mu_{2}+\beta\right) X}}{\left(\mu_{2}+\beta\right)} \\
& +\frac{\left(t_{2}+t_{4}\right)}{\left(s_{2} \mu_{2}-\lambda\right)} \frac{1-e^{-\left(s_{2} \mu_{2}-\lambda+\beta\right) X}}{\left(s_{2} \mu_{2}-\lambda+\beta\right)}-\frac{\left(t_{4}-t_{3}\right)}{\left(s_{1} \mu_{1}-\lambda\right)} \frac{1-e^{-\left(s_{1} \mu_{1}-\lambda+\beta\right) X}}{\left(s_{1} \mu_{1}-\lambda+\beta\right)}
\end{aligned}
$$

The expected value for tardiness can be calculated based on equations (3) and (11) as:

$$
\begin{aligned}
& E[C]=E[I]+E[W]-\int_{0}^{X}\left(1-F_{L}(\tau)\right) d \tau \\
& =\left(\frac{\left(t_{1}+t_{2}\right)}{\mu_{1}}+\frac{\left(t_{3}-t_{1}\right)}{\mu_{2}}-\frac{\left(t_{2}+t_{4}\right)}{\left(s_{2} \mu_{2}-\lambda\right)}+\frac{\left(t_{4}-t_{3}\right)}{\left(s_{1} \mu_{1}-\lambda\right)}-1\right) \frac{1-e^{-\beta X}}{\beta} \\
& -\frac{\left(t_{1}+t_{2}\right)}{\mu_{1}} \frac{1-e^{-\left(\mu_{1}+\beta\right) X}}{\left(\mu_{1}+\beta\right)}-\frac{\left(t_{3}-t_{1}\right)}{\mu_{2}} \frac{1-e^{-\left(\mu_{2}+\beta\right) X}}{\left(\mu_{2}+\beta\right)} \\
& +\frac{\left(t_{2}+t_{4}\right)}{\left(s_{2} \mu_{2}-\lambda\right)} \frac{1-e^{-\left(s_{2} \mu_{2}-\lambda+\beta\right) X}}{\left(s_{2} \mu_{2}-\lambda+\beta\right)}-\frac{\left(t_{4}-t_{3}\right)}{\left(s_{1} \mu_{1}-\lambda\right)} \frac{1-e^{-\left(s_{1} \mu_{1}-\lambda+\beta\right) X}}{\left(s_{1} \mu_{1}-\lambda+\beta\right)} \\
& +\frac{\left(m_{1}+o_{1}\right)}{\mu_{1}^{2}}-\frac{o_{1}}{\left(\left(1-\rho_{1}\right) s_{1} \mu_{1}\right)^{2}}+\frac{\left(m_{2}+o_{2}\right)}{\mu_{2}^{2}}-\frac{o_{2}}{\left(\left(1-\rho_{2}\right) s_{1} \mu_{2}\right)^{2}}
\end{aligned}
$$

In Figure 2 WIP, FGI lead time, and tardiness are shown to illustrate their dependency on utilization, number of machines, and processing rates of machines.

Figures 2a, 2c, and 2e show the influence of a different number of machines at stages 1 and 2. The number of machines at each stage is predefined and the processing rate is varied. The influence of predefined processing rates is depicted in Figures $2 b$, $2 \mathrm{~d}$, and $2 \mathrm{f}$. The processing rate is kept equal at both stages and the number of machines is varied.

The results from Figure 2a, 2c, and $2 \mathrm{e}$ show that an imbalance between the processing rates at the two stages which creates an imbalance in number of machines, leads to an increase in WIP, a decrease in FGI lead time and an increase in tardiness at the same utilization. Figure $2 \mathrm{~b}, 2 \mathrm{~d}$, and $2 \mathrm{f}$ show that whenever the processing rate is predefined, contrary to Figures $2 \mathrm{a}, 2 \mathrm{c}$, and $2 \mathrm{e}$ only certain utilization rates are 
possible. In the examples shown in Figure 2b, 2d, and 2f, especially high utilization values cannot be reached because of the relation between processing rate and order arrival rate. Nevertheless, higher processing rates lead to lower WIP, higher FGI lead time, and lower tardiness values at the same utilization.

Insert Figure 2 about here.

For the optimization problem (6) minimizing the FGI, backorder, WIP, and capacity costs when a set of processing rates are predefined, only the problem statement can be provided in explicit form; the solution has to be found numerically. For given capacity investment, Altendorfer and Minner, (2010) provide the following solution for optimal work ahead window:

$$
\begin{aligned}
& X^{*}: F_{\tilde{W}_{n}}(X)=\frac{c_{c}}{c_{f}+c_{c}} \\
& \Leftrightarrow \frac{c_{c}}{c_{f}+c_{c}}=\left(t_{1}+t_{2}\right) \frac{1-e^{-\mu_{1} X}}{\mu_{1}}+\left(t_{3}-t_{1}\right) \frac{1-e^{-\mu_{2} X}}{\mu_{2}} \\
& -\left(t_{2}+t_{4}\right) \frac{1-e^{-\left(s_{2} \mu_{2}-\lambda\right) X}}{\left(s_{2} \mu_{2}-\lambda\right)}+\left(t_{4}-t_{3}\right) \frac{1-e^{-\left(s_{1} \mu_{1}-\lambda\right) X}}{\left(s_{1} \mu_{1}-\lambda\right)}
\end{aligned}
$$

4.2. Solution heuristic for the two-stage $M / M / s$ optimization problem In this section we propose a simple solution algorithm for problem (6) based on the two-stage M/M/1 problem and on Proposition 4. In the numerical example provided in the following section it is shown that this simple algorithm leads to good results when being compared to an enumeration scheme for finding the optimal solution.

\section{Algorithm}

Step 1: Calculate $\mu_{1}^{*}$ and $\mu_{2}^{*}$ of the two-stage M/M/1 problem with continuous processing rates (see Altendorfer and Minner, 2010). 


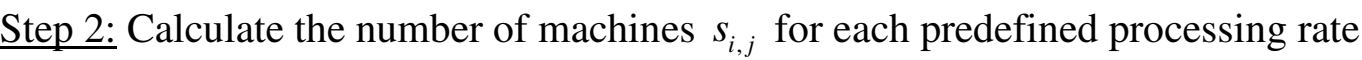
$\mu_{j} \in \Omega$ and for both stages $i \in\{1,2\}$ by $\left.s_{i, j}=\max \left(\left\lceil\frac{\lambda}{\mu_{j}}\right\rceil ; \mid \frac{\mu_{i}^{*}}{\mu_{j}}\right]\right)$.

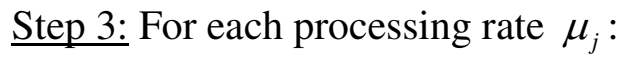

Calculate the cost term $C\left(s_{1, j}, \mu_{j}, s_{2}^{*}, \mu_{2}^{*}, X^{*}\left(s_{1, j}, \mu_{j}, s_{2}^{*}, \mu_{2}^{*}\right)\right)$, whereby $s_{2}^{*}=1$ from $\mu_{2}^{*}$ being calculated in the single machine case.

If $C\left(s_{1, j}+1, \mu_{j}, s_{2}^{*}, \mu_{2}^{*}, X^{*}\left(s_{1, j}+1, \mu_{j}, s_{2}^{*}, \mu_{2}^{*}\right)\right)<C\left(s_{1, j}, \mu_{j}, s_{2}^{*}, \mu_{2}^{*}, X^{*}\left(s_{1, j}, \mu_{j}, s_{2}^{*}, \mu_{2}^{*}\right)\right)$ then increase $s_{1, j}$ until

$$
\begin{aligned}
& C\left(s_{1, j}+1, \mu_{j}, s_{2}^{*}, \mu_{2}^{*}, X^{*}\left(s_{1, j}+1, \mu_{j}, s_{2}^{*}, \mu_{2}^{*}\right)\right)> \\
& C\left(s_{1, j}, \mu_{j}, s_{2}^{*}, \mu_{2}^{*}, X^{*}\left(s_{1, j}, \mu_{j}, s_{2}^{*}, \mu_{2}^{*}\right)\right)
\end{aligned}
$$

is fulfilled and otherwise decrease $s_{1, j}$ until

$$
\begin{aligned}
& C\left(s_{1, j}-1, \mu_{j}, s_{2}^{*}, \mu_{2}^{*}, X^{*}\left(s_{1, j}-1, \mu_{j}, s_{2}^{*}, \mu_{2}^{*}\right)\right)> \\
& C\left(s_{1, j}, \mu_{j}, s_{2}^{*}, \mu_{2}^{*}, X^{*}\left(s_{1, j}, \mu_{j}, s_{2}^{*}, \mu_{2}^{*}\right)\right)
\end{aligned}
$$

is fulfilled and set $s_{1, j}^{*}=s_{1, j}$.

Select the values $\left\{s_{1, j}^{*}, \mu_{1}^{*}(\Omega)\right\}$ minimizing the cost function. $X^{*}$ is implicitly defined in equation (13).

Step 4: Redo the same procedure as in Step 3 for $C\left(s_{1, j}^{*}, \mu_{1}^{*}(\Omega), s_{2, j}, \mu_{j}, X^{*}\left(s_{1, j}^{*}, \mu_{1}^{*}(\Omega), s_{2, j}, \mu_{j}\right)\right)$ whereby $s_{2, j}$ is increased/decreased and $\left\{s_{2, j}^{*}, \mu_{2}^{*}(\Omega)\right\}$ is selected

Two limiting assumptions are made in this solution heuristic. The first assumption is that Proposition 4 holds for any cost structure. The second assumption is that it is sufficient to optimize the number of machines and processing rates stagewise. When optimizing stage 1 , the parameters for stage 2 are set to one machine with 
$\mu_{2}^{*}$ whereas when stage 2 is optimized, the optimal parameters for stage 1 from Step 3 are applied. Additionally, an efficient starting point for the optimal capacity search is included in the algorithm. Note that also all combinations of optimality candidates on both stages could be tested but this would lead to a considerable additional computational effort.

\section{Numerical example}

\subsection{Influence of machine size on optimal cost}

The influence of different processing rates on the optimal costs for a production system with balanced capacities $\left(\mu_{1}=\mu_{2}\right)$ is investigated. The balanced case is chosen since the main observations are not influenced by that assumption but it reduces complexity. Table 1 shows the parameters for the four cases compared in this section. Case $\mathrm{A}$ is the basic scenario. Case $\mathrm{B}$ is a situation where capacity costs are low, in Case $\mathrm{C}$ the mean customer required lead time is very short and in Case D the tardiness penalties are very high.

Insert Table 1 about here.

Insert Figure 3 about here.

For these four cases the optimal costs and the optimal number of machines are numerically determined by solving the optimization problem (6) for processing rate values between 0.001 and 1.2. The calculation has been performed in Wolfram Mathematica 6.0. Figure 3 presents the results. The following observations can be made.

Observation 1: Whenever different machine sizes with different processing rates are predefined and only the work ahead window and the number of machines can be 
optimized, the optimal number of machines is highly dependent on the predetermined processing rates.

This observation illustrates the trade-off between economies of scale (recommending a single machine) and the requirement of capacity coordination between stages. It shows that for practical applications it is not always optimal to invest into only a single machine capable of handling all the customer demands. A lot of capacity can be wasted by this one big machine instead of having several smaller machines.

Observation 2: The range of optimal costs is a convex function with respect to the predefined processing rates. Very big and very small machines induce a high risk of incurring too high costs.

This statement is based on the difference between the highest and the lowest optimal costs when a certain range of processing rates is focussed. In Case A, the highest and lowest costs for a processing rate range between 1.0 and 1.2 is 1650 whereas it is only 715 for a processing rate range of 0.4 to 0.6 and it increases again to $\infty$ for a processing rate range of 0 to 0.2 . Assuming a predetermined set of available processing rates, Observation 2 states that also small machines can fit with a high probability. This observation is supported by the results from Section 5.3 where 1875 test instances are compared applying 4 processing rate sets. In $38 \%$ of the cases more than one machine is optimal at least at one of the two stages.

The result for Case B shows that the range of optimal costs as a function of the predefined processing rate reduces when capacity costs are lower. The Cases C and D confirm that the observations are robust to changes in mean customer required lead time and backorder costs. 
Figure 4 shows the optimal number of machines for two different machine size policies. Parameters of Case A are used and the vertical black lines show the available processing rates.

Insert Figure 4 about here.

In Figure 4a, we assume three different machine sizes defined by three different available processing rates. In this case the smallest machine (lowest processing rate) leads to the lowest overall costs, which is consistent with Observation 2. In Figure 4b, there are 6 processing rates available. In this case the optimal processing rate is neither the smallest nor the largest one. As the example from Figure 4 shows, the optimal number of machines highly depends on the machine sizes available. This is true although the overall minimum is always found with one machine per stage when the processing rate can be continuously scaled as stated in Proposition 1.

Comparing Observations 1 and 2 with the TPS (Toyota Production System) philosophy to invest into small and simple machines shows that this philosophy can lead to a cost premium paid which is, however, smaller than expected based on Proposition 1.

\subsection{Influence of machine number on optimal costs}

For a set of test instances the optimal processing rate is determined for a predefined machine number. Therefore, we solve problem (6) under condition (13) with continuous processing rates for the balanced case (equations are provided in the Appendix). The test instances consist of all combinations of the following parameter sets: $1 / \beta \in\{12,36,60,84,108\}, \lambda=1, c_{\mu, 1}=c_{\mu, 2}=c_{\mu} \in\{100+225 k, k=0, \ldots, 4\}$, $c_{c} \in\{10+5 k, k=0, \ldots, 4\}, c_{y, 1} \in\{2 k, k=1, \ldots, 5\}, c_{y, 2} \in\{2 k, k=1, \ldots, 5\} \leq c_{y, 1}$. The 
Insert Figure 5 about here.

\subsection{Performance evaluation of solution heuristic}

In this section the same problem instances as described in Section 5.2 are used. Four different processing rate sets are tested: $\mu_{i}($ Case 1$) \in\left\{1 / 10 * 2^{k}, k=0, \ldots, 4\right\}$, $\mu_{i}($ Case 2$) \in\left\{1 / 7.5^{*} 2^{k}, k=0, \ldots, 4\right\}, \mu_{i}($ Case 3$) \in\left\{1 / 6^{*} k, k=1, \ldots, 9\right\}$, and $\mu_{i}($ Case 4$) \in\{1 / 4 * k, k=1, \ldots, 6\}$. From Figure 4 , we conjecture that not only the number of values in a set of possible processing rates, but also their basic value, has a significant influence on costs. Therefore, two different $\mu$-value schemes each with two different basic values are tested.

For the overall 1875 test instances the optimal solution with continuous range of processing rates, the optimal solution applying the four processing rate sets, and the heuristic solution from Section 4.2 have been calculated. The optimal solution is found by enumeration of processing rates and number of machines under condition (13). In the current numerical example which is programmed in Wolfram Mathematica 6.0, the computation time reduction with the heuristic is $90 \%$. 
Insert Table 2 about here.

The results in Table 2 indicate that the solution heuristic leads to good results. Only in a few cases the solution found deviates from the optimal one, but the average cost increase is negligible.

\subsection{Influence of uncertain input rates}

In real investment problems the customer order rate, i.e. the parameter $\lambda$, is uncertain at the time of investment. We assume that prior to the capacity investment decision only uncertain information about the input rate is available. In particular, the customer order rate follows a three point distribution with a low, medium and high expected input rate $\lambda_{l}, \lambda_{m}$, and $\lambda_{h}$ occurring with probabilities $P\left(\lambda_{l}\right), P\left(\lambda_{m}\right)$, and $P\left(\lambda_{h}\right)$ respectively. After the investment the real input rate is observed and the optimal work ahead window is determined. For the two-stage case with equal processing rates at both stages and a continuous range of processing rates the optimization problem is:

$$
\begin{aligned}
& P\left(\lambda_{l}\right) C\left(X_{l}, \mu, s, \lambda_{l}\right)+P\left(\lambda_{m}\right) C\left(X_{m}, \mu, s, \lambda_{m}\right) \\
& +P\left(\lambda_{h}\right) C\left(X_{h}, \mu, s, \lambda_{h}\right) \rightarrow \min _{X_{l}, X_{m}, X_{h}, \mu, s}
\end{aligned}
$$

$C(\cdot)$ is the cost function from equation (6) with a continuous range of processing rates.

The numerical results in Figure 6 and Figure 7 illustrate the influence of the range between $\lambda_{l}$ and $\lambda_{h}$, and the influence of the order rate probabilities $P\left(\lambda_{l}\right)$ and $P\left(\lambda_{h}\right)$ in comparison to $P\left(\lambda_{m}\right)$. All cost factors are taken from Case A and the range parameters are shown in Table 3:

Insert Table 3 about here. 
Insert Figure 6 about here.

Insert Figure 7 about here.

Observation 3: The uncertainty in input rates has no influence on the single machine optimality property stated in Proposition 1. Uncertain input rates do not favour the investment of more than one machine if processing rates can be chosen continuously. In this case, the cost functions with respect to the processing rate look similar to the ones presented in Figures 3 and 4.

Observation 4: Arrival rate parameter uncertainty leads to a considerable increase in optimal costs and the optimal capacity invested increases.

Observation 4 is intuitive as variability increases when uncertainty of input rate increases and this variability increase has to be covered with additional capacity and additional costs for inventory and backorders. Even though this finding is linked in the current example to a symmetric structure in uncertainty we conjecture that an asymmetric uncertainty has no influence on that result in general. Furthermore, if the high input rate realizes, no possibility to reduce the input rate by rejecting customers is included in this setting which could be a field of further research.

A second experiment with a limited capacity decision postponement option is conducted for a predefined set of processing rates. When $\lambda$ is uncertain, the technology, i.e. the machine size has to be decided. After observing the true customer order input rate, additional machines with the same processing rate can be added. This leads to the following optimization problem:

$$
\begin{aligned}
& P\left(\lambda_{l}\right) C\left(X_{l}, \mu, s_{l}, \lambda_{l}\right)+P\left(\lambda_{m}\right) C\left(X_{m}, \mu, s_{m}, \lambda_{m}\right) \\
& +P\left(\lambda_{h}\right) C\left(X_{h}, \mu, s_{h}, \lambda_{h}\right) \rightarrow \min _{X_{l}, X_{m}, X_{h}, \mu, s_{l}, s_{m}, s_{h}}
\end{aligned}
$$


The optimization problem is solved for the test instanced from Section 5.3 with the simplification that a balanced production system is considered and that $c_{y, 1}=c_{y, 2}$. This leads to 625 test instances which are compared for the four processing rate sets and $P\left(\lambda_{l}\right)=0.25 ; P\left(\lambda_{m}\right)=0.50 ; P\left(\lambda_{h}\right)=0.25$. The percentages of test instances where more than 1 machine is optimal are shown in Table 4:

Insert Table 4 about here.

Observation 5: An increase in range uncertainty leads to a higher probability that more than 1 machine (with smaller processing rates) are optimal in comparison to a known customer order rate when only the machine type (processing rate) has to be decided under uncertainty.

For most of the processing rate sets, demand rate uncertainty leads to an increase of instances where more than 1 machine, however with a lower processing rate, is optimal. As a managerial insight, under demand rate uncertainty it is beneficial to invest into smaller machines if investment into additional machines of the same type is possible after observing the real customer order input rate.

\section{Conclusion}

We analyzed the influence of machine size on the optimal costs for WIP, FGI, backorders, and capacity. A general optimization problem for an $n$-stage production system is stated and explicit expressions for expected WIP, FGI, and backorders are developed for a two-stage M/M/s production system with exponentially distributed customer required lead time and a work ahead window order release policy.

For the $n$-stage production system a general condition for single machine optimality at each stage is provided and it is shown that in a system with value added 


\section{References}

Altendorfer K, Jodlbauer H. 2010. An analytical model for service level and tardiness in a single machine MTO production system. Forthcoming: International Journal of Production Research. DOI 10.1080/00207541003660176

Altendorfer K, Minner S. Simultaneous Capacity and Planned Lead Time Optimization for a two-stage Production System. Working paper, FH-Steyr

Angelus A, Porteus EL. 2002. Simultaneous Capacity and Production Management of Short-Life-Cycle, Produce-to-Stock Goods Under Stochastic Demand. Management Science;48; 399-413.

Bertrand JWM, van Ooijen HPG. 2008. Optimal work order release for make-to-order job shops with customer order lead-time costs, tardiness costs and work-in-process costs. International Journal of Production Economics;116; 233-241.

Bradley JR, Glynn PW. 2002. Managing Capacity and Inventory Jointly in Manufacturing Systems. Management Science;48; 273-288.

Brigham G. 1955. On a congestion problem in an aircraft factory. Journal of the Operations Research Society of America; 3; 412-428.

Brumelle SL. 1971. Some inequalities for parallel-server queues. Operations Research;19; 402-413.

Buzacott JA, Shanthikumar JG. 1992. Design of manufacturing systems using queueing models. Queueing Systems;12; 135-213. 
Buzacott JA, Shanthikumar JG. 1994. Safety Stock Versus Safety Time in MRP Controlled Production Systems. Management Science;40; 1678-1689.

Chao X, Scott C. 2000. Several results on the design of queueing systems. Operations Research;48; 965-970.

Crabill TB, Gross D, Magazine MJ. 1977 A Classified Bibliography of Research on Optimal Design and Control of Queques. Operations Research;25; 219-232.

Driscoll MF, Weiss NA. 1976. An application of queueing theory to reservation networks. Management Science;22; 540-546.

Grassmann W. 1983. The Convexity of the Mean Queue Size of the M/M/c Queue with Respect to the Traffic Intensity. Journal of Applied Probability;20; 916-919.

Hillier FS. 1963. Economic models for industrial waiting line problems. Management Science; $10 ; 119-130$.

Hopp WJ, Spearman ML. 1996. Factory Physics:. Foundation of Manufacturing Management. Irwin: Chicago.

Jodlbauer H. 2008. Customer driven production planning. International Journal of Production Economics; $111 ; 793-801$.

Jodlbauer H, Altendorfer K. 2010. Trade-off between capacity invested and inventory needed. European Journal of Operational Research;203; 118-133.

MacGregor-Smith J. 2007. Multi-server, Finite Waiting Room, M / G / c / K Optimization Models. INFOR;45; 257-274.

Mandelbaum A, Reiman MI. 1998. On Pooling in Queueing Networks. Management Science;44; 971-981.

Medhi J. 1991. Stochastic Models in Queuing Theory. MA: Academic Press: Boston.

Mincsovics G, Tan T, Alp O. 2009. Integrated capacity and inventory management with capacity acquisition lead times. European Journal of Operational Research;196; 949-958.

Raman A, Kim B. 2002. Quantifying the Impact of Inventory Holding Cost and Reactive Capacity on an Apparel Manufacturer's Profitability. Production \& Operations Management; $11 ; 358-373$.

Rao SS, Gunasekaran A, Goyal SK, Martikainen T. 1998. Waiting line model applications in manufacturing. International Journal of Production Economics;54; $1-28$.

Scheller-Wolf A. 2003. Necessary and sufficient conditions for delay moments in FIFO multiserver queues with an application comparing s slow servers with one fast one. Operations Research;51; 748-758.

Silver EA, Pyke DF, Peterson R. 1998. Inventory Management and Production Planning and Scheduling. $3^{\text {rd }}$ ed. John Wiley and Sons: New York.

Stidham S, Jr. 1970. On the optimality of single server queuing systems. Operations Research;18; 708-732.

Stidham S, Jr. 1992. Pricing and capacity decisions for service facility: stability and multiple local optima. Management Science;38; 1121-1139.

Tijms HC. 2003. A First Course in Stochastic Models. Wiley: Amsterdam.

Yano CA. 1987. Setting Planned Leadtimes in Serial Production Systems with Tardiness Costs. Management Science;33; 95-106.

Zipkin PH. 2000. Foundations of Inventory Management. McGraw-Hill Higher Education: Boston, a. o. 


\section{Appendix A - Proofs}

\section{Proof of Proposition 1}

For any multi-machine production system with parameters $\left(\left\{c_{i}\right\} ;\left\{\mu_{i}\right\}\right)$, a single machine production system with the same capacity costs and parameters $\left(\{1\} ;\left\{\mu_{i}^{1}\right\}\right)$ and $\mu_{i}^{1}=c_{i} \mu_{i}$ exists. The superscript 1 indicates the system with a single machine at each stage. The WIP, FGI, and backorder costs in the single-machine system have to be smaller than in the parallel machine system:

$$
\begin{aligned}
& \sum_{i=1}^{n} E\left[\tilde{W}_{i}\right] \lambda\left(c_{y, i}-c_{y, i+1}\right)+E\left[\tilde{W}_{n}\right] \lambda c_{c}+E[I] \lambda\left(c_{f}+c_{c}\right) \\
& -X\left(1-F_{L}(X)\right) \lambda c_{c}+\sum_{i=1}^{n} c_{i} \mu_{i} c_{\mu, i}>\sum_{i=1}^{n} E\left[\tilde{W}_{i}^{1}\right] \lambda\left(c_{y, i}-c_{y, i+1}\right) \\
& +E\left[\tilde{W}_{n}^{1}\right] \lambda c_{c}+E\left[I^{1}\right] \lambda\left(c_{f}+c_{c}\right)-X\left(1-F_{L}(X)\right) \lambda c_{c}+\sum_{i=1}^{n} \mu_{i}^{1} c_{\mu, i} \\
& \Leftrightarrow \sum_{i=1}^{n} E\left[\tilde{W}_{i}\right] \lambda\left(c_{y, i}-c_{y, i+1}\right)+E\left[\tilde{W}_{n}\right] \lambda c_{c}+E[I] \lambda\left(c_{f}+c_{c}\right)> \\
& \sum_{i=1}^{n} E\left[\tilde{W}_{i}^{1}\right] \lambda\left(c_{y, i}-c_{y, i+1}\right)+E\left[\tilde{W}_{n}^{1}\right] \lambda c_{c}+E\left[I^{1}\right] \lambda\left(c_{f}+c_{c}\right) \\
& \Leftrightarrow E\left[\tilde{W}_{i}\right]>E\left[\tilde{W}_{i}^{1}\right] \forall i \text { and } E[I]>E\left[I^{1}\right]
\end{aligned}
$$

Condition $E\left[\tilde{W}_{i}\right]>E\left[\tilde{W}_{i}^{1}\right]$ is fulfilled with condition (5). It remains to show that $E[I]>E\left[I^{1}\right]$ holds. Applying equation (2) leads to:

$$
\begin{aligned}
& E[I]>E\left[I^{1}\right] \\
& \Leftrightarrow \int_{0}^{X} \int_{0}^{\theta} f_{\tilde{W}_{n}}(\tau)(\theta-\tau) d \tau f_{L}(\theta) d \theta+\int_{X}^{\infty} \int_{0}^{X} f_{\tilde{W}_{n}}(\tau)(X-\tau) d \tau f_{L}(\theta) d \theta> \\
& \int_{0}^{X} \int_{0}^{\theta} f_{\tilde{W}_{n}^{1}}(\tau)(\theta-\tau) d \tau f_{L}(\theta) d \theta+\int_{X}^{\infty} \int_{0}^{X} f_{\tilde{W}_{n}^{1}}(\tau)(X-\tau) d \tau f_{L}(\theta) d \theta \\
& \Leftrightarrow \int_{0}^{X}\left(\int_{0}^{\theta} f_{\tilde{W}_{n}}(\tau)(\theta-\tau) d \tau-\int_{0}^{\theta} f_{\tilde{W}_{n}^{1}}(\tau)(\theta-\tau) d \tau\right) f_{L}(\theta) d \theta \\
& +\int_{X}^{\infty}\left(\int_{0}^{X} f_{\tilde{W}_{n}}(\tau)(X-\tau) d \tau-\int_{0}^{X} f_{\tilde{W}_{n}^{1}}(\tau)(X-\tau) d \tau\right) f_{L}(\theta) d \theta>0
\end{aligned}
$$

by partial integration it follows: 


$$
\begin{aligned}
& \left.\Leftrightarrow F_{\tilde{W}_{n}}(\tau)(\theta-\tau)\right|_{0} ^{\theta}-\int_{0}^{\theta} F_{\tilde{W}_{n}}(\tau) d \tau>\left.F_{\tilde{W}_{n}^{1}}(\tau)(\theta-\tau)\right|_{0} ^{\theta}-\int_{0}^{\theta} F_{\tilde{W}_{n}^{1}}(\tau) d \tau \forall \theta>0 \\
& \Leftrightarrow \int_{0}^{\theta} F_{\tilde{W}_{n}^{1}}(\tau)-F_{\tilde{W}_{n}}(\tau) d \tau \geq 0 \forall \theta>0 \text { and } \exists \theta: \int_{0}^{\theta} F_{\tilde{W}_{n}^{1}}(\tau)-F_{\tilde{W}_{n}}(\tau) d \tau>0
\end{aligned}
$$

which is the definition of second order stochastic dominance.

\section{Proof of Proposition 2}

Assume a set of different processing rates in a manufacturing system $\{\mu(1) \geq \mu(2) \geq \ldots \geq \mu(n)\}$. From the structure of equations (2) to (4) it follows that backorder and FGI costs are independent of the sequence in which these processing rates are allocated to the stages. Therefore, these costs can be omitted for the proof. Comparing any unordered system, e.g. $\mu_{1}=\mu(2)<\mu_{2}=\mu(1)$ and $\left\{\mu_{3}=\mu(3), \mu_{4}=\mu(4), \ldots, \mu_{n}=\mu(n)\right\}$ to the corresponding ordered system $\left\{\mu_{1}=\mu(1) \geq \mu_{2}=\mu(2) \geq \ldots \geq \mu_{n}=\mu(n)\right\}$, leads to the following cost inequality:

$$
C_{\text {unordered }} \stackrel{!}{>} C_{\text {ordered }}
$$

with

$$
\begin{aligned}
& C_{\text {unordered }}: \mu_{1}=\mu(2)<\mu_{2}=\mu(1) \text { and }\left\{\mu_{3}=\mu(3), \mu_{4}=\mu(4), \ldots, \mu_{n}=\mu(n)\right\} \\
& C_{\text {ordered }}:\left\{\mu_{1}=\mu(1), \mu_{2}=\mu(2), \ldots, \mu_{n}=\mu(n)\right\} \\
& \Leftrightarrow E\left[W_{1}(\mu(2))\right] \lambda c_{y, 1}+E\left[W_{2}(\mu(1))\right] \lambda c_{y, 2}+\xi(\mu(2)) c_{\mu, 1}+\xi(\mu(1)) c_{\mu, 2} \\
& \vdots E\left[W_{1}(\mu(1))\right] \lambda c_{y, 1}+E\left[W_{2}(\mu(2))\right] \lambda c_{y, 2}+\xi(\mu(1)) c_{\mu, 1}+\xi(\mu(2)) c_{\mu, 2} \\
& \Leftrightarrow
\end{aligned}
$$

1) $\frac{\xi(\mu(1))}{\xi(\mu(2))}\left(c_{\mu, 2}-c_{\mu, 1}\right)>c_{\mu, 2}-c_{\mu, 1}$; fulfilled with $\mu(1)>\mu(2)$ and $c_{\mu, 2} \geq c_{\mu, 1}$

2) $\left(E\left[W_{1}(\mu(2))\right]-E\left[W_{1}(\mu(1))\right]\right) \frac{c_{y, 1}}{c_{y, 2}}>E\left[W_{2}(\mu(2))\right]-E\left[W_{2}(\mu(1))\right]$ fulfilled with $E\left[W_{1}(\mu(2))\right]-E\left[W_{1}(\mu(1))\right]=E\left[W_{2}(\mu(2))\right]-E\left[W_{2}(\mu(1))\right]$ and $c_{y, 1} \geq c_{y, 2}$ 


\section{Proof of Proposition 4}

Restating the costs from equation (1) applying equation (3) leads to:

$$
\begin{aligned}
& C\left(X,\left\{s_{i}\right\},\left\{\mu_{i}\right\}\right)=\sum_{i=1}^{n} E\left[W_{i}\left(s_{i}, \mu_{i}\right)\right] \lambda\left(c_{y, i}+c_{c}\right) \\
& +E\left[I\left(X,\left\{s_{i}\right\},\left\{\mu_{i}\right\}\right)\right] \lambda\left(c_{f}+c_{c}\right)-\int_{0}^{X}\left(1-F_{L}(\tau)\right) d \tau \lambda c_{c}+\sum_{i=1}^{n} s_{i} \mu_{i} c_{\mu, i}
\end{aligned}
$$

For any $s_{k}=\tilde{s}$ from $\left\{s_{i}\right\}$ the following cost delta when increasing $\tilde{s}$ by 1 follows:

$$
\begin{aligned}
& \Delta C(\tilde{s}, \cdot)=C\left(X,\left\{s_{i} \neq s_{k}\right\}, \tilde{s}+1,\left\{\mu_{i}\right\}\right)-C\left(X,\left\{s_{i} \neq s_{k}\right\}, \tilde{s},\left\{\mu_{i}\right\}\right)= \\
& \left(E\left[W_{k}\left(\tilde{s}+1, \mu_{k}\right)\right]-E\left[W_{k}\left(\tilde{s}, \mu_{k}\right)\right]\right) \lambda\left(c_{y, k}+c_{c}\right) \\
& +\left(E\left[I\left(X,\left\{s_{i} \neq s_{k}\right\}, \tilde{s}+1,\left\{\mu_{i}\right\}\right)\right]-E\left[I\left(X,\left\{s_{i} \neq s_{k}\right\}, \tilde{s},\left\{\mu_{i}\right\}\right)\right]\right) \lambda\left(c_{f}+c_{c}\right) \\
& +\mu_{k} c_{\mu, k}
\end{aligned}
$$

which can be rewritten as:

$$
\begin{aligned}
& \Delta C(\tilde{s}, \cdot)=\left(E\left[W_{k}(\tilde{s}+1)\right]-E\left[W_{k}(\tilde{s})\right]\right) \lambda\left(c_{y, k}+c_{c}\right) \\
& +\left(E\left[I\left(\left\{s_{i} \neq s_{k}\right\}, \tilde{s}+1\right)\right]-E\left[I\left(\left\{s_{i} \neq s_{k}\right\}, \tilde{s}\right)\right]\right) \lambda\left(c_{f}+c_{c}\right)+\mu_{k} c_{\mu, k} \\
& =\left(\int_{0}^{\infty} \tau f_{W}(\tau, \tilde{s}+1, \cdot) d \tau-\int_{0}^{\infty} \tau f_{W}(\tau, \tilde{s}, \cdot) d \tau\right) \lambda\left(c_{y, k}+c_{c}\right) \\
& +\left(\int_{0}^{X} F_{W}(\tau,(\tilde{s}+1, \cdot))\left(1-F_{L}(\tau)\right) d \tau\right. \\
& \left.-\int_{0}^{X} F_{W}(\tau,(\tilde{s}, \cdot))\left(1-F_{L}(\tau)\right) d \tau\right) \lambda\left(c_{f}+c_{c}\right)+\mu_{k} c_{\mu, k} \\
& =-\int_{0}^{\infty}\left(F_{W}(\tau, \tilde{s}+1, \cdot)-F_{W}(\tau, \tilde{s}, \cdot)\right) d \tau \lambda\left(c_{y, k}+c_{c}\right) \\
& +\int_{0}^{X} F_{W}(\tau,(\tilde{s}+1, \cdot))-F_{W}(\tau,(\tilde{s}, \cdot))\left(1-F_{L}(\tau)\right) d \tau \lambda\left(c_{f}+c_{c}\right)+\mu_{k} c_{\mu, k}
\end{aligned}
$$

with $c_{y, k}=c_{f}=c_{y}$

$$
\begin{aligned}
& =-\int_{0}^{\infty}\left(F_{W}(\tau, \tilde{s}+1, \cdot)-F_{W}(\tau, \tilde{s}, \cdot)\right) d \tau \lambda\left(c_{y}+c_{c}\right) \\
& +\int_{0}^{X} F_{W}(\tau,(\tilde{s}+1, \cdot))-F_{W}(\tau,(\tilde{s}, \cdot))\left(1-F_{L}(\tau)\right) d \tau \lambda\left(c_{y}+c_{c}\right)+\mu_{k} c_{\mu, k}
\end{aligned}
$$

which decreases in $\tilde{s}$ since $E\left[W_{k}\left(s_{k}, \mu_{k}\right)\right]$ decreases in $s_{k}$. For an M/M/s queue this property is shown by Grassmann, (1983) who proves a convex decrease of 
$E\left[W_{k}\left(s_{k}, \mu_{k}\right)\right]$ with respect to $s_{k}$. However, it holds for any production system, since an additional machine can never increase production lead time and whenever the additional machine processes a job while others are waiting, the waiting time of the other jobs decreases. The serial setting of more than one processing stage does not change this property.

\section{Derivation of expressions for two-stage $M / M /$ s production system with identical} processing rates and identical number of machines

For the calculations in Section 5, the expressions for expected WIP, FGI, and

backorders are derived for a two-stage $\mathrm{M} / \mathrm{M} / \mathrm{s}$ production system with equal

processing rates and equal number of machines at both stages.

With $\mu_{1}=\mu_{2}=\mu, s_{1}=s_{2}=s, \rho_{1}=\rho_{2}=\rho$ it follows $m_{1}=m_{2}=m, o_{1}=o_{2}=o$

and the pdf of the overall production lead time is:

$$
\begin{aligned}
& f_{W_{\text {balaneed }}}(t)=\int_{0}^{t}\left((m+o)^{2} e^{-\mu t}-(m+o) o e^{(-s \mu+\lambda) t+(s \mu-\lambda-\mu) \tau}\right. \\
& \left.-(m+o) o e^{-\mu t+(\mu-s \mu+\lambda) \tau}+o^{2} e^{(\lambda-s \mu) t}\right) d \tau \\
& =(m+o)^{2} t e^{-\mu t}+o^{2} t e^{-(s \mu-\lambda) t}-2(m+o) o \frac{e^{-(s \mu-\lambda) t}-e^{-\mu t}}{(\mu-s \mu+\lambda)}
\end{aligned}
$$

Based on the pdf of the overall production lead time its cdf is:

$$
\begin{aligned}
& F_{W}(\tau)=\int_{0}^{\tau} f_{W}(\theta) d \theta=(m+o)^{2} \frac{1-e^{-\mu \tau}(1+\mu \tau)}{\mu^{2}}+o^{2} \frac{1-e^{-(s \mu-\lambda) \tau}(1+(s \mu-\lambda) \tau)}{(s \mu-\lambda)^{2}} \\
& -2(m+o) o \frac{1-e^{-(s \mu-\lambda) \tau}}{(\mu-s \mu+\lambda)(s \mu-\lambda)}+2(m+o) o \frac{1-e^{-\mu \tau}}{(\mu-s \mu+\lambda) \mu}
\end{aligned}
$$

From equation (5) and equation (2) the expected FGI lead time is: 


$$
\begin{aligned}
& E\left[I_{\text {balanced }}\right]=\int_{0}^{X} F_{W}(\tau)\left(1-F_{L}(\tau)\right) d \tau \\
& =\left(\frac{(m+o)^{2}}{\mu^{2}}+\frac{o^{2}}{(s \mu-\lambda)^{2}}-\frac{2(m+o) o}{(\mu-s \mu+\lambda)(s \mu-\lambda)}+\frac{2(m+o) o}{(\mu-s \mu+\lambda) \mu}\right) \frac{1-e^{-\beta X}}{\beta} \\
& -\left(\frac{(m+o)^{2}}{\mu^{2}}+\frac{2(m+o) o}{(\mu-s \mu+\lambda) \mu}\right) \frac{1-e^{-(\mu+\beta) X}}{(\mu+\beta)}-\frac{(m+o)^{2}}{\mu} \frac{1-e^{-(\mu+\beta) X}}{(\mu+\beta)^{2}} \\
& +\frac{(m+o)^{2}}{\mu} \frac{e^{-(\mu+\beta) X} X}{(\mu+\beta)}-\frac{o^{2}}{(s \mu-\lambda)} \frac{1-e^{-(s \mu-\lambda+\beta) X}(1+(s \mu-\lambda+\beta) X)}{(s \mu-\lambda+\beta)^{2}} \\
& +\left(\frac{2(m+o) o}{(\mu-s \mu+\lambda)(s \mu-\lambda)}-\frac{o^{2}}{(s \mu-\lambda)^{2}}\right) \frac{1-e^{-(s \mu-\lambda+\beta) X}}{(s \mu-\lambda+\beta)}
\end{aligned}
$$

and the optimality condition (13) becomes

$$
\begin{aligned}
& X^{*}: \frac{c_{c}}{c_{f}+c_{c}}=(m+o)^{2} \frac{1-e^{-\mu \tau}(1+\mu \tau)}{\mu^{2}}+o^{2} \frac{1-e^{-(s \mu-\lambda) \tau}(1+(s \mu-\lambda) \tau)}{(s \mu-\lambda)^{2}} \\
& -2(m+o) o \frac{1-e^{-(s \mu-\lambda) \tau}}{(\mu-s \mu+\lambda)(s \mu-\lambda)}+2(m+o) o \frac{1-e^{-\mu \tau}}{(\mu-s \mu+\lambda) \mu}
\end{aligned}
$$

\section{Appendix B - List of Variables}

The following Table 5 provides a summary of all variables.

Insert Table 5 about here. 


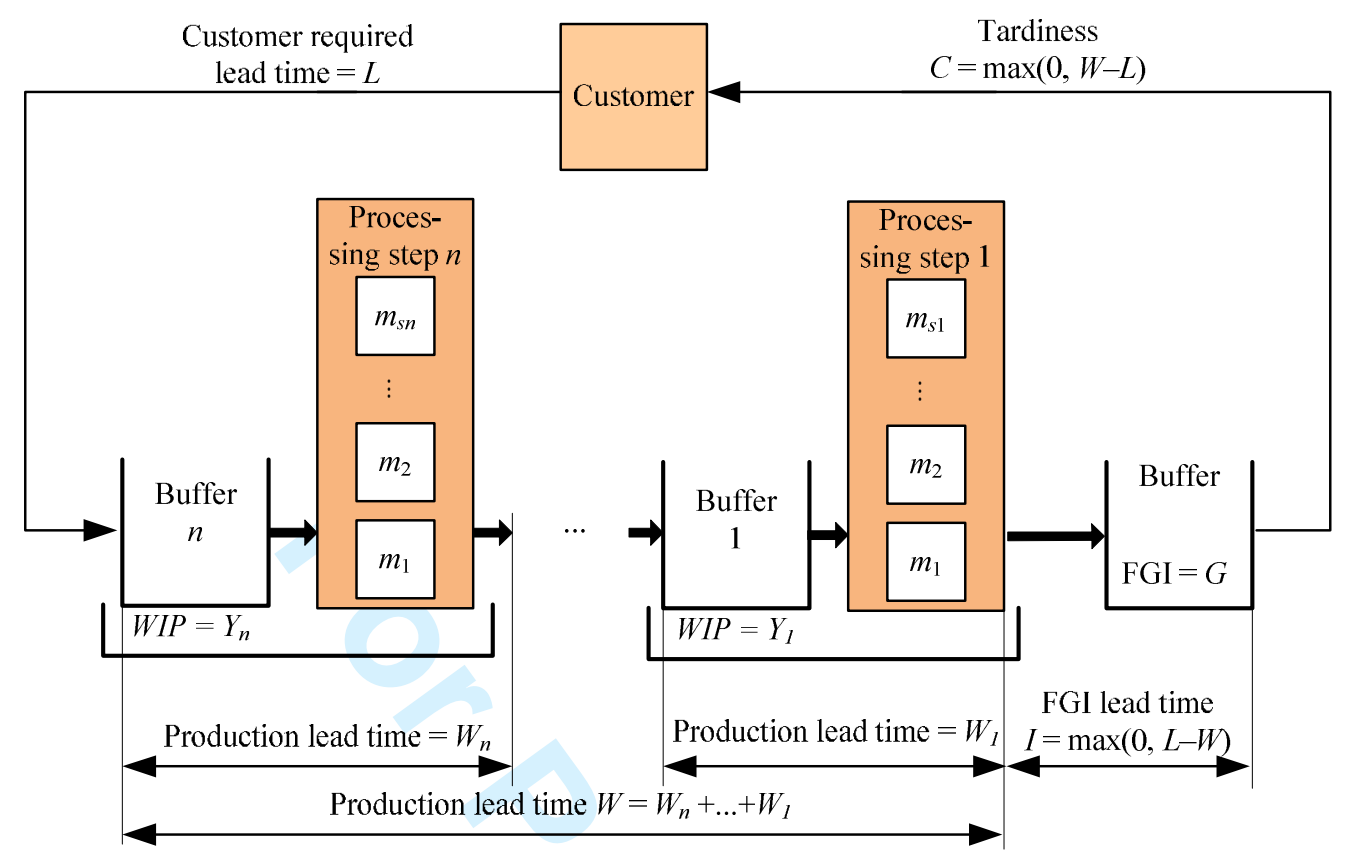

Figure 1. Production system. 

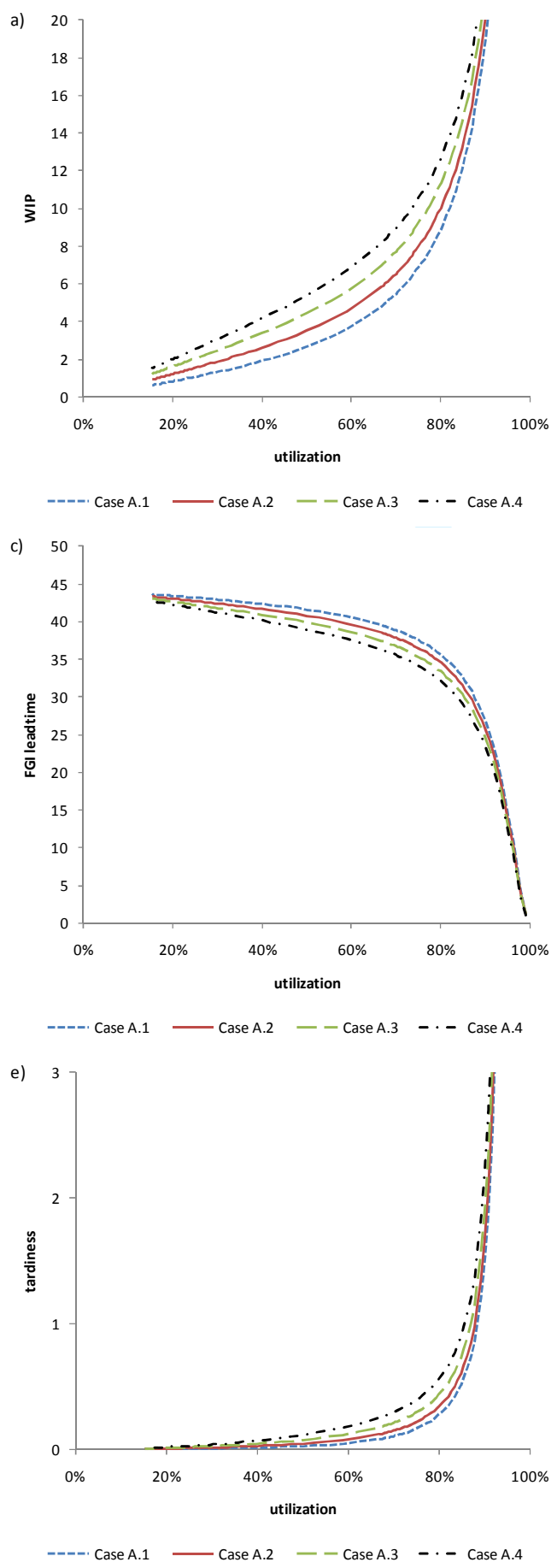

$\lambda=1 ; \quad X=20 ; \beta=0.005 ; \mathrm{s}_{1}=2$;

$s_{2}($ Case A. 1$)=2 ; s_{2}($ Case A.2) $=4$;

$s_{2}($ Case A.3 $)=6 ; s_{2}($ Case A.4 $)=8$;

$\mu_{i}=\lambda /\left(s_{i} \rho\right)$
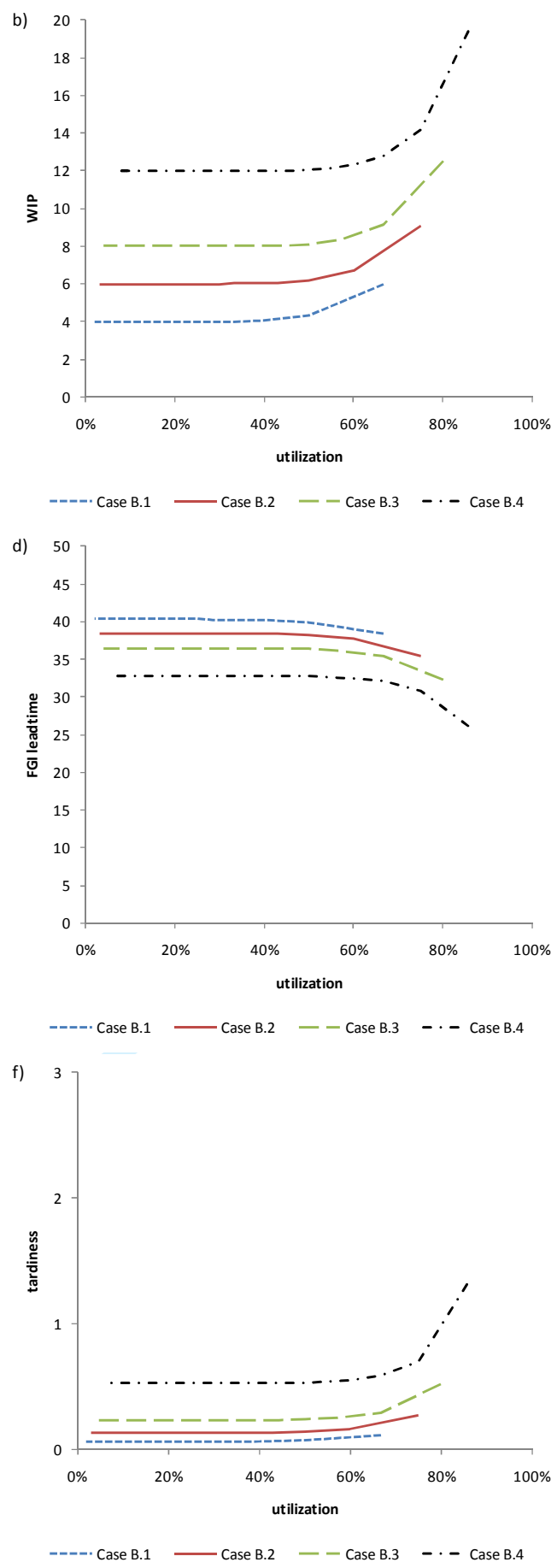

$\lambda=1 ; \quad X=20 ; \beta=0.005$

$\mu($ Case B. 1$)=0.5 ; \mu($ Case B. 2$)=0 . \overline{3}$;

$\mu($ Case B. 3$)=0.25 ; \mu($ Case B. 4$)=0.1 \overline{6}$ $s_{i}=\left\lceil\lambda /\left(\mu_{i} \rho\right)\right\rceil ;$

Figure 2. Influence of processing rate and number of machines on WIP, FGI lead time and tardiness. 


\begin{tabular}{|l|r|r|r|r|r|l|l|}
\hline Case & $c_{c}$ & $c_{f}$ & $c_{y, 2}$ & $c_{y, 1}$ & $c_{\mu, 1}=c_{\mu, 2}$ & $\lambda$ & $\beta$ \\
\hline $\mathrm{A}$ & 30 & 10 & 5 & 7.5 & 1,000 & 1 & 0.005 \\
\hline $\mathrm{B}$ & 30 & 10 & 5 & 7.5 & 250 & 1 & 0.005 \\
\hline $\mathrm{C}$ & 30 & 10 & 5 & 7.5 & 1,000 & 1 & 0.05 \\
\hline $\mathrm{D}$ & 100 & 10 & 5 & 7.5 & 1,000 & 1 & 0.005 \\
\hline
\end{tabular}

Table 1. Test cases
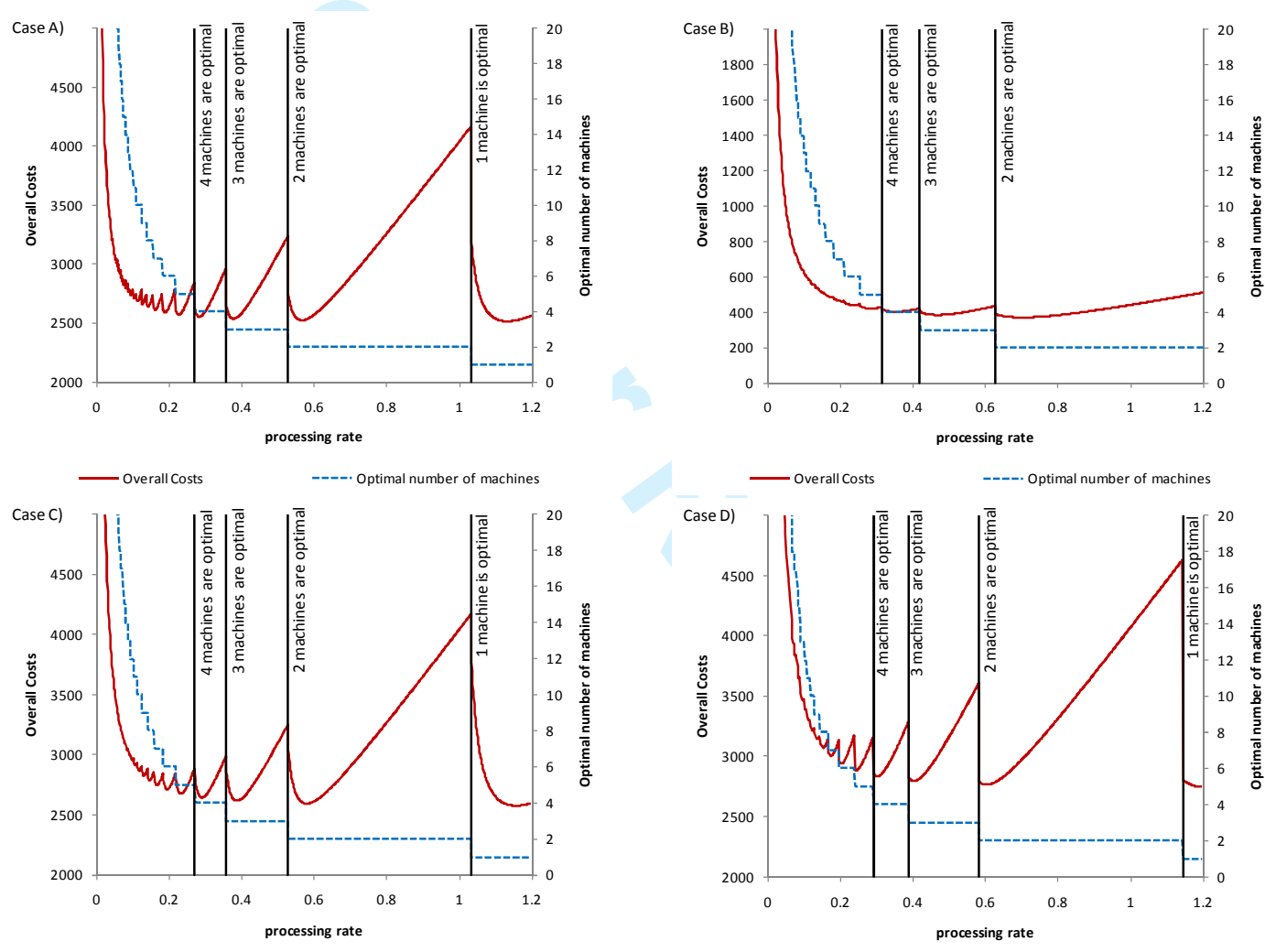

Figure 3. Optimal overall costs dependent on machine size.
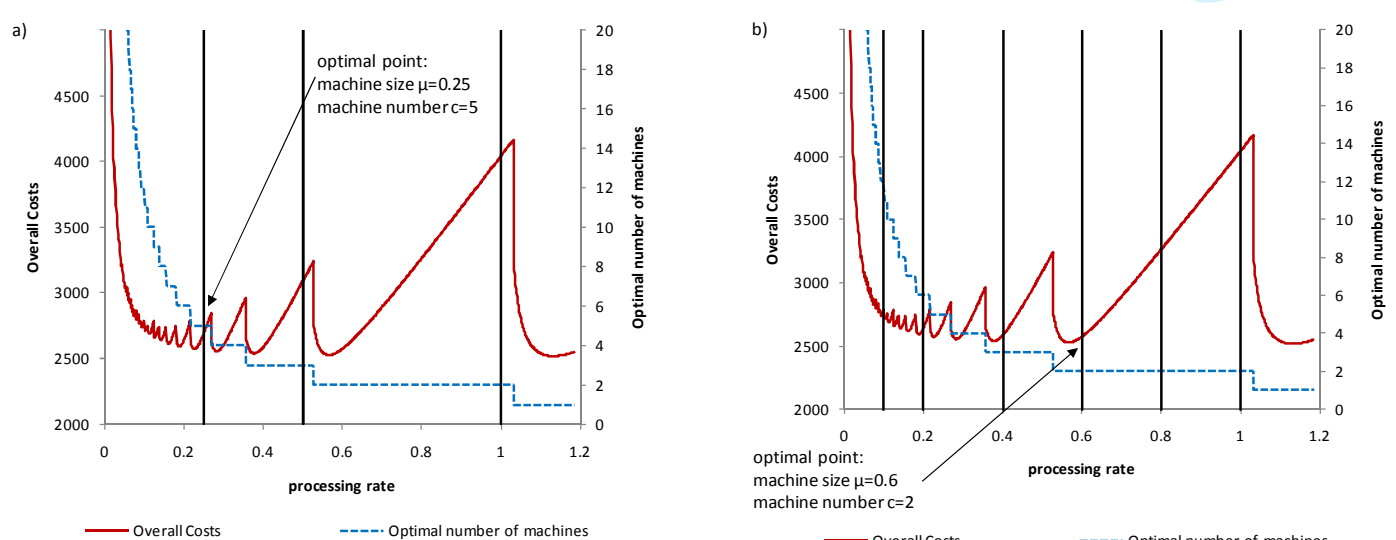


\begin{tabular}{|l|l|l|l|}
\hline $\begin{array}{l}\text { Processing rate } \\
\text { set }\end{array}$ & $\begin{array}{l}\text { Avg. cost increase } \\
\text { by proc. rate set }\end{array}$ & $\begin{array}{l}\text { \% of test instances } \\
\text { heuristic is optimal }\end{array}$ & $\begin{array}{l}\text { Avg. cost increase } \\
\text { by heuristic }\end{array}$ \\
\hline Case 1 & $3.2 \%$ & $100 \%$ & $0 \%$ \\
\hline Case 2 & $8.4 \%$ & $95.9 \%$ & $0.006 \%$ \\
\hline Case 3 & $0.6 \%$ & $99.1 \%$ & $0.001 \%$ \\
\hline Case 4 & $2.6 \%$ & $99.7 \%$ & $0.0003 \%$ \\
\hline
\end{tabular}

Table 2. Performance comparison.

\begin{tabular}{|l|c|c|c|}
\hline Case & $\lambda_{l}$ & $\lambda_{m}$ & $\lambda_{h}$ \\
\hline Low range & 0.95 & 1 & 1.05 \\
\hline Medium range & 0.90 & 1 & 1.10 \\
\hline High range & 0.80 & 1 & 1.20 \\
\hline
\end{tabular}

Table 3. Range uncertainty values.

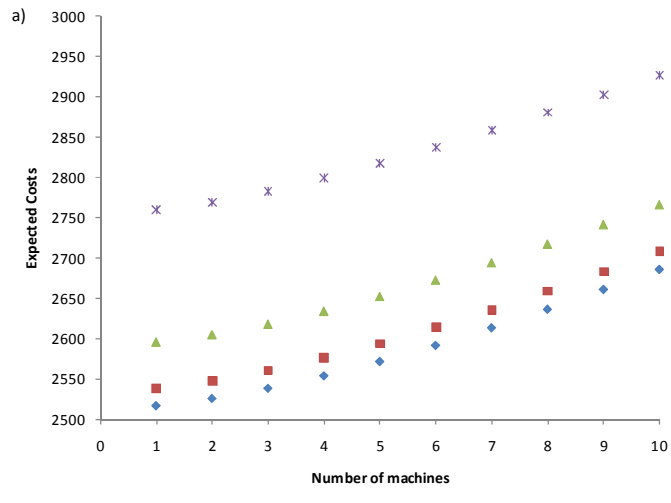

- constant $\|$ low range $\Delta$ medium range $*$ high range

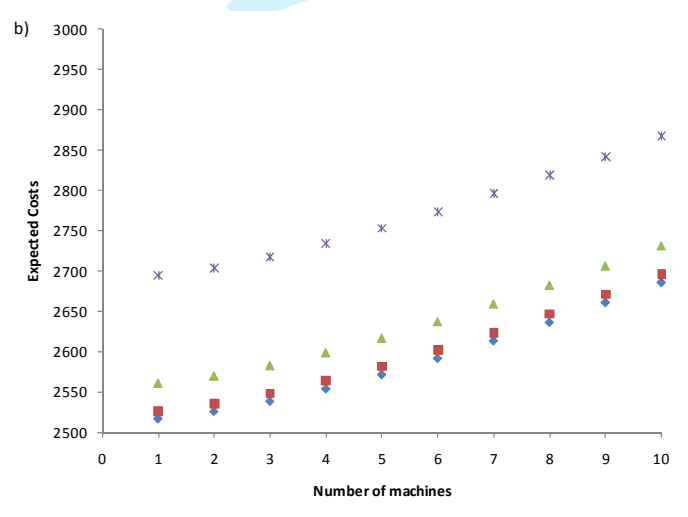

- constant - low range $\Delta$ medium range $*$ highrange

$$
P\left(\lambda_{l}\right)=0.25 ; P\left(\lambda_{m}\right)=0.50 ; P\left(\lambda_{h}\right)=0.25 \quad P\left(\lambda_{l}\right)=0.1 ; P\left(\lambda_{m}\right)=0.8 ; P\left(\lambda_{h}\right)=0.1
$$

Figure 6. Influence of uncertain input rate information on optimal costs. 

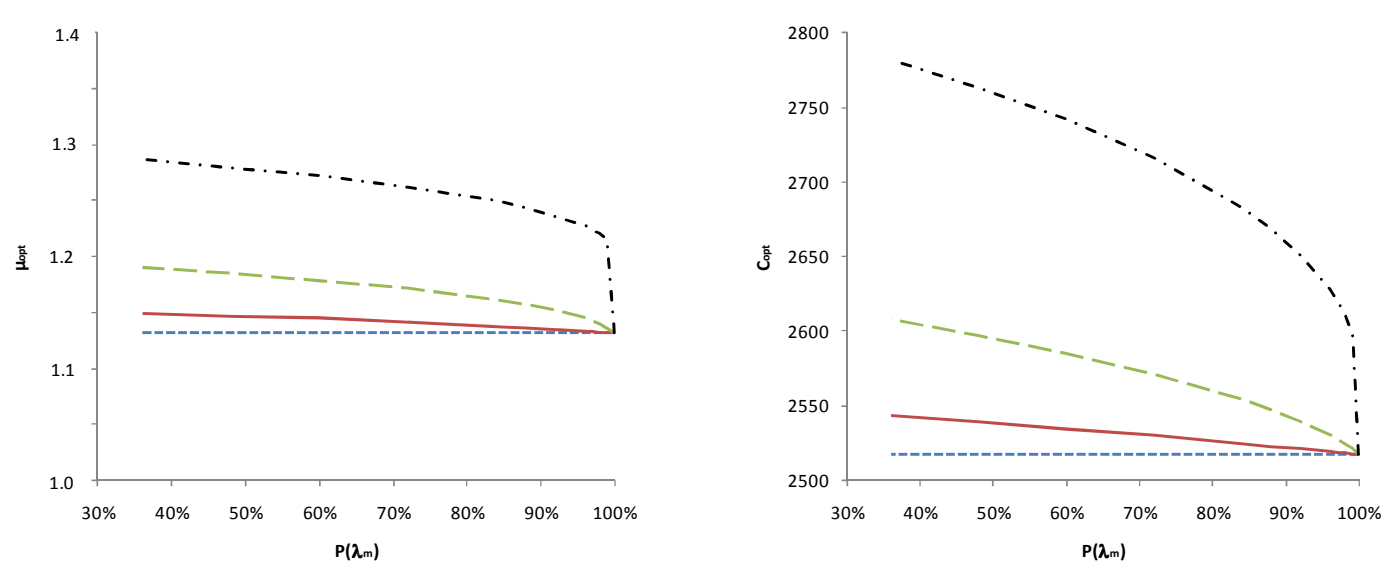

----- constant $\_$low range -- medium range $-\cdot-$ high range

----- constant $\_$low range -- medium range $-\cdot-$ high range

$$
P\left(\lambda_{l}\right)=P\left(\lambda_{h}\right)=\frac{1-P\left(\lambda_{m}\right)}{2}
$$

Figure 7. Influence of uncertain input rate on processing rate and costs.

\begin{tabular}{|l|c|c|c|c|c|c|c|c|}
\hline \multirow{2}{*}{} & \multicolumn{3}{|c|}{$\begin{array}{c}\text { \% of cases where more than 1 } \\
\text { machine is optimal }\end{array}$} & \multicolumn{5}{c|}{$\begin{array}{c}\text { average processing rate of machines } \\
\text { invested }\end{array}$} \\
\cline { 2 - 10 } & Case 1 & Case 2 & Case 3 & Case 4 & Case 1 & Case 2 & Case 3 & Case 4 \\
\hline Known $\lambda$ & $81.9 \%$ & $65.9 \%$ & $0.0 \%$ & $0.0 \%$ & 0.61 & 0.57 & 1.22 & 1.29 \\
\hline Low variability in $\lambda$ & $81.8 \%$ & $100.0 \%$ & $0.0 \%$ & $2.6 \%$ & 0.62 & 0.31 & 1.23 & 1.26 \\
\hline Medium variability in $\lambda$ & $82.2 \%$ & $100.0 \%$ & $1.6 \%$ & $23.5 \%$ & 0.58 & 0.32 & 1.24 & 1.06 \\
\hline High variability in $\lambda$ & $82.6 \%$ & $100.0 \%$ & $69.4 \%$ & $81.3 \%$ & 0.49 & 0.32 & 0.61 & 0.50 \\
\hline
\end{tabular}

Table 4. Range uncertainty - optimality of more than 1 machine. 
Table 5. List of Variables.

random variable, cdf, and pdf of production lead time at stage $i$

$f_{W_{i}}(\cdot)$

respectively

$\tilde{W}_{i}, F_{\tilde{W}_{i}}(\cdot), \quad$ random variable, cdf, and pdf of remaining production lead time

$f_{\tilde{W}_{i}}(\cdot)$ from arrival at stage $i$ until its delivery to the FGI buffer respectively

random variable of WIP at stage $i$

random variable of FGI lead time

random variable of FGI

random variable of tardiness

$B$

random variable of backorders

$L, F_{L}(\cdot), f_{L}(\cdot)$

random variable, cdf, and pdf of customer required lead time respectively

$\beta$

parameter of the customer required lead time distribution ( $1 / \beta$ is the mean customer required lead time)

$\mu_{i} \quad$ processing rate at stage $i$

$\Omega$

set of predefined processing rates

$\lambda$

order arrival rate

$\lambda_{l}, \lambda_{m}, \lambda_{h}$

$X$

$c_{y, i}$

$c_{f}$

$c_{c}$

$c_{\mu, i}$

$s_{i}$

$p_{0}$

$\rho_{i}$

$C(\cdot)$

$m, o$,

$t_{1}, t_{2}, t_{3}, t_{4}$
Low, medium, and high value for expected customer order arrival rate when demand is uncertain

work ahead window

WIP holding costs at stage $i$ for one order being stored one period of time

FGI holding costs for 1 order being stored 1 period of time

backorder costs for one order being late one period of time

capacity costs for one unit of capacity invested at stage $i$

number of machines at stage $i$

probability that no order is in the production system for $\mathrm{M} / \mathrm{M} / \mathrm{s}$ queue

utilization of stage $i$

costs to be minimized

auxiliary variables in the two-stage $\mathrm{M} / \mathrm{M} / \mathrm{s}$ model for simplification of statements 
